\title{
Intrinsic Self-Healing Process in Cementitious Materials
}

\author{
MO LI ${ }^{1}$ AND SHUAI FAN ${ }^{1}$ \\ 1Department of Civil and Environmental Engineering, 4145 \\ Engineering Gateway, University of California, Irvine, \\ CA 92697-2175. Email: moli@uci.edu
}

Designing self-healing capacity into cementitious materials can open a new world of opportunities for more durable and resilient concrete infrastructure. The intrinsic self-healing approach relies on the healing potential naturally possessed by cementitious materials, through carbonation of portlandite and continued hydration of unreacted clinkers. However, a fundamental question remains as to how robust this intrinsic self-healing process is. Depite that healing has been observed from crack top surface, the healing extent along crack depth remains much less known. Little is understood about the mechanisms driving the intrinsic healing process along the crack depth, and the effects of different physical and chemical conditions on the healing efficiency below the crack surface. These questions are critical for understanding the crack healing potential within cementitious materials under various conditions.

This study focuses on understanding the intrinsic healing process along crack depth in cementitious materials with various physical and chemical parameters. A new, coupled transport-thermodynamics model is formulated to predict crack wall profile change with time due to healing. The model considers the time-dependent interaction among ions transport through crack depth, healing products precipitation along crack walls, and local alteration of crack transport properties. The model was then validated by controlled experiments quantifying the 3D crack aperture field before and after healing. Using X-ray computed microtomography, scanning electron microscopy and chemical analysis, the experiments uncovered and quantified the non-homogenous healing ratios and chemical compositions along crack depth. Parametric studies further elucidated how different physical or chemical parameters affect the healing profile change with time. The results shed light on the intrinsic healing mechanisms and robustness in cementitious materials. 